Sequential Threat Detection for Harbor Defense: An X-ray Physics-Based Bayesian Approach

J. V. Candy

March 21, 2013

IEEE OCEANS'13

Bergen, Norway June 10, 2013 through June 14, 2013 
This document was prepared as an account of work sponsored by an agency of the United States government. Neither the United States government nor Lawrence Livermore National Security, LLC, nor any of their employees makes any warranty, expressed or implied, or assumes any legal liability or responsibility for the accuracy, completeness, or usefulness of any information, apparatus, product, or process disclosed, or represents that its use would not infringe privately owned rights. Reference herein to any specific commercial product, process, or service by trade name, trademark, manufacturer, or otherwise does not necessarily constitute or imply its endorsement, recommendation, or favoring by the United States government or Lawrence Livermore National Security, LLC. The views and opinions of authors expressed herein do not necessarily state or reflect those of the United States government or Lawrence Livermore National Security, LLC, and shall not be used for advertising or product endorsement purposes. 


\title{
Sequential Threat Detection for Harbor Defense: An X-ray Physics-Based Bayesian Approach
}

\author{
J. V. Candy, Fellow, IEEE
}

\begin{abstract}
The timely and accurate detection of threat contraband especially for ports-of-entry (e.g. harbors, bays, borders, airports) is an extremely critical problem of national security. The investigation of advanced techniques to reliably and accurately detect threats and reject non-threats is the major focus of this effort. The characterization of signal processing models based on $x$ ray transport physics is a crucial element in advanced sequential Bayesian processor designs. Incorporating the underlying statistics of x-ray interactions with materials offering a potentially unique signature of an object or item under investigation leads to a (stochastic) physics-based approach. State-space models, common in many application areas, are introduced into the $x$-ray radiation area. Here the resulting processor incorporating this construct is developed from a pragmatic perspective. A Gaussian application is discussed to illustrate feasibility of the overall physics-based approach. It is shown that the sequential Bayesian processor is capable of providing a reliable and accurate solution with high confidence in a timely manner for this problem based on a set of synthesized object intensity data.
\end{abstract}

\section{INTRODUCTION}

Countries throughout the world rely heavily on transporting goods daily on ships and aircraft to maintain stable economies. Screening for threat/contraband items in containers (cargo, shipping containers, baggage, etc.) is the primary function of detection systems deployed in airports and other points-ofentry especially at shipping/receiving ports. The timely and accurate detection of threats is an extremely critical problem of national security. The utilization of high-energy x-rays enables data that can be used for threat and contraband detection. The development of advanced techniques to reliably and accurately detect threats and reject non-threats is the major focus of homeland security for many nations. Thus, the basic problem is to detect the presence of threat items (e.g. explosives) concealed in shipping containers cargo, etc.

One of the major obstacles from a commercial perspective is that these threats can easily be concealed with items that occupy the same container. Currently, x-ray scanners are employed to detect the presence or absence of a threat, but may be degraded by a variety of uncertainties generated by the hardware, noise and any uncertainties created by the interaction of x-rays with these items [1]-[3]. Processing raw $\mathrm{x}$-ray images of the scanned container requires innovative methods for detection. It is therefore necessary to develop customized techniques to scan a container and sort through the variety of items to decide whether or not a threat is present.

The characterization of signal processing models based on x-ray transport physics is a crucial element in advanced sequential Bayesian processor designs. Incorporating the underlying statistics of $\mathrm{x}$-ray interactions with materials offers a potentially unique signature of an object or item under investigation leading to a (stochastic) physics-based approach. State-space models, common in many application areas, are introduced into the $\mathrm{x}$-ray propagation. Here the resulting processor incorporating this construct is developed from a pragmatic perspective. A Gaussian application is discussed to illustrate feasibility of the overall physics-based approach. It is shown that the Bayesian processor is capable of providing a reliable and accurate solution with high confidence in a timely manner for this problem for a set of synthesized $\mathrm{x}$-ray intensity data of an object.

In this paper we develop a novel representation evolving from the usual $x$-ray transport physics (Lambert-Beer law)the state-space model. State-space is a well-known, general characterization of many dynamic systems incorporating a wealth of theoretical and practical results. We first briefly discuss the underlying transport physics and then introduce the state-space models for a lumped model approach providing a potential solution to the detection problem. Next we develop a state-space $\mathrm{x}$-ray transport simulator showing the applicability of these techniques to synthesize both deterministic as well as stochastic (Gauss-Markov) transport. The sequential Bayesian threat detection processor is developed next following the Wald sequential probability ratio test applied to this problem. Here we develop the generic processor for our problem and then develop a physics-based solution for the Gaussian case. Based on the lumped model simulation, we apply the sequential Bayesian detection scheme to synthesized data demonstrating its capability. The results are summarized and future work discussed in the final section.

The basic approach is model-based incorporating the $\mathrm{x}$-ray propagation physics into the state-space framework. That is, the initial polyenergetic source intensity is measured at the output of a detector array (multi-output). These data along with a-priori knowledge of the targeted threat are incorporated into a threat model with the appropriate attenuation coefficient parameters. Next, measured object intensities are estimated from the raw detector data using a linear model-based processor $(M B P)$ or Kalman filter to complete the log-likelihood decision function. Then at each step (spatial), the intensity is predicted by the MBP and a decision (threat/non-threat/no decision) is made. Based on the Lambert-Beer law and the state-space representation, the sequential log-likelihood ratio detector is illustrated below in principle and application (Fig. 1). The plots illustrate the processor as well as the application to synthesized threat data.

In this paper we first develop the transport physics and 
then introduce the state-space models for a "lumped model' approach in Sec. 2. Next we develop a state-space x-ray trans port simulator showing the applicability of these technique to synthesize both deterministic as well as stochastic (Gauss Markov) transport in Sec. 3. Sequential Bayesian threat detec tion is developed in Sec. 4 following the Wald sequential prob ability ratio test applied to this problem. Here we develop th generic processor for our problem and then develop a solution for the Gaussian case. Based on the lumped model simulation we apply the sequential Bayesian detection scheme to synthe sized data (ammonium-nitrate explosive threat) demonstratin: its capability. The results are summarized and future worl discussed in the final section.

\section{PHYSICS-BASED MODELS}

The characterization of a wealth of items that could br enclosed in a shipping container covers a wide range $o$ features. From an x-ray interrogation perspective for threa detection, we are concerned primarily with the interaction o $\mathrm{x}$-ray photons (energy) with the variety of materials enclosed within.

\section{A. Background: X-ray Physics}

When $\mathrm{x}$-rays interact with matter (materials), they undergo attenuation effects, that is, an x-ray or equivalently beam of photons passing through matter is depleted or attenuated as photons are removed through absorption and scattering [1], [2]. Here photon energy $(\epsilon)$ is transformed to heat (absorption) or scattered with its energy dissipated and re-directed. Assume we have a perfectly parallel monoenergetic (single energy) beam of x-rays with intensity $\mathcal{I}(\epsilon, x)$ irradiating a uniformly thick, single material absorber as shown in Fig. 1, then the transmitted (output) intensity is governed by

$$
\frac{d}{d x} \mathcal{I}(\epsilon, x)=-\mu(\epsilon, \mathcal{Z}, \rho) \mathcal{I}(\epsilon, x)
$$

where $\mu(\epsilon, \mathcal{Z}, \rho)$ is the attenuation coefficient $\left(\mathrm{cm}^{-1}\right)$ at energy $\epsilon(\mathrm{KeV})$ for a "single" material of mass density $\rho$ $\left(\frac{g}{c m^{3}}\right)$ with atomic number $\mathcal{Z}$ along the $x$-direction of path length $\triangle x=x-x_{o}(\mathrm{~cm})$ with $\mathcal{I}\left(\epsilon_{o}, x_{o}\right)$ is the initial intensity. Solving this equation leads to the Lambert-Beer Law [1], [2] given by

$$
\mathcal{I}(\epsilon, x)=\mathcal{I}\left(\epsilon_{o}, x_{o}\right) \times \exp \left\{-\mu(\epsilon, \mathcal{Z}, \rho)\left(x-x_{o}\right)\right\}
$$

This relation can be extended further to incorporate composite materials $\left((\mathcal{Z}, \rho) \longrightarrow\left(\mathcal{Z}_{m}, \rho_{m}\right) ; m=1, \cdots, M\right)$ and multiple source energies ( $S\left(\epsilon_{n}\right) ; n=1, \cdots, N$ ) giving the general expression

$$
\begin{aligned}
& \mathcal{I}\left(S\left(\epsilon_{n}\right), x\right)=\mathcal{I}\left(S\left(\epsilon_{o}\right), x_{o}\right) \\
\times & \exp \left\{-\int\left(\sum_{m=1}^{M} \mu_{m}\left(S\left(\epsilon_{n}\right), \mathcal{Z}_{m} \rho_{m}\right) \triangle x\right) d \epsilon\right\}
\end{aligned}
$$

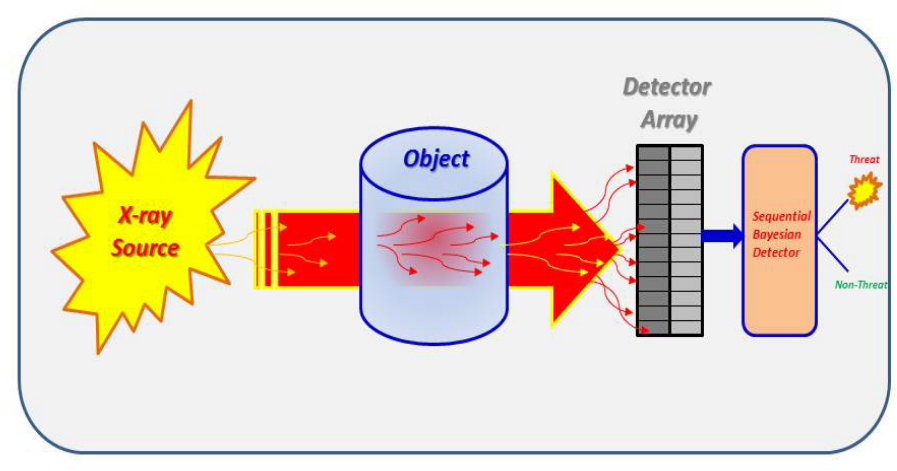

Fig. 1. X-ray Transmission Physics: polyenergetic source, object, detector array measurements and sequential Bayesian processing for threat detection.

for $n=1, \cdots, N$.

From these results, we see that the attenuation coefficients contain all of the critical physical information required to uniquely characterize the material under irradiation by the source X-rays.

\section{B. Physics-Based State-Space (Lumped) Models}

1) X-ray Transmission: State-space representations of dynamic systems have long been recognized as a solution to various classes of differential/difference equations. Following their introduction to a variety of technical areas, an enormous amount of systems theory has evolved enabling the development generic algorithms that merely require the modelers to transform their models into state-space form enabling them to be incorporated into this powerful framework [9]. Once accomplished many properties can be analyzed and exploited to solve a variety of problems. State-space models are very useful for physics applications and can be placed in a convenient vector-matrix form useful for signal processing, estimation and detection.

For our problem, we return to the original first order differential transmission relation of Eq. 1 and realize that it is already in the well-known state-space form [9]. We consider this model a "lumped model" where the object is characterized by a set of average attenuation coefficients. Assuming that a polyenergetic source consisting of a set of discrete monoenergetic energies, $\epsilon_{n} ; n=1, \cdots, N$, irradiates the material (object), Beer's law is now a set of first order differential transport equations, that is,

$$
\frac{d}{d x} \mathcal{I}\left(\epsilon_{n}, x\right)=-\mu\left(\epsilon_{n}, \mathcal{Z}, \rho\right) \mathcal{I}\left(\epsilon_{n}, x\right) ; n=1, \cdots, N
$$


with incident intensities given by $\mathcal{I}\left(\epsilon_{n o}, x_{n o}\right)$ identifying each monoenergetic source component. Expanding over $n$ we obtain the equivalent state-space transmission model given by

$$
\begin{gathered}
\frac{d}{d x}\left[\begin{array}{c}
\mathcal{I}\left(\epsilon_{1}, x\right) \\
\vdots \\
\mathcal{I}\left(\epsilon_{N}, x\right)
\end{array}\right]= \\
{\left[\begin{array}{ccc}
-\mu\left(\epsilon_{1}, \mathcal{Z}, \rho\right) & & O \\
O & \ddots & -\mu\left(\epsilon_{N}, \mathcal{Z}, \rho\right)
\end{array}\right]\left[\begin{array}{c}
\mathcal{I}\left(\epsilon_{1}, x\right) \\
\vdots \\
\mathcal{I}\left(\epsilon_{N}, x\right)
\end{array}\right]}
\end{gathered}
$$

which can be expressed simply in vector-matrix form as

$$
\frac{d}{d x} \underline{\mathcal{I}}(\epsilon, x)=A(\epsilon, \mathcal{Z}, \rho) \underline{\mathcal{I}}(\epsilon, x)
$$

where we have the state intensity vector $\underline{\mathcal{I}} \in \mathcal{R}^{N \times 1}$, the system (physics) matrix, $A \in \mathcal{R}^{N \times N}$ with initial (incident) intensity vector defined by

$$
\underline{\mathcal{I}}\left(\epsilon_{o}, x_{o}\right):=\left[\mathcal{I}\left(\epsilon_{1 o}, x_{1 o}\right), \cdots, \mathcal{I}\left(\epsilon_{N o}, x_{N o}\right)\right]^{T}
$$

and ${ }^{T}$ the matrix transpose operator. It is well-known that the solution to the linear shift invariant ( $A$ not a function of $\mathcal{I}$ ) state equations is given by the state transition matrix as [9]

$$
\mathcal{T}(\epsilon, x)=e^{A(\epsilon, \mathcal{Z}, \rho)\left(x-x_{o}\right)}
$$

with $\mathcal{T} \in \mathcal{R}^{N \times N}$ yielding Beer's law for a polyenergetic source constructed as a composition of monoenergetic sources, that is,

$$
\underline{\mathcal{I}}(\epsilon, x)=\mathcal{T}(\epsilon, x) \underline{\mathcal{I}}\left(\epsilon_{o}, x\right)=e^{A(\epsilon, \mathcal{Z}, \rho)\left(x-x_{o}\right)} \underline{\mathcal{I}}\left(\epsilon_{o}, x_{o}\right)
$$

This representation can be considered a "lumped model" where the object is characterized by a set of average attenuation coefficients $\left\{\mu\left(\epsilon_{n}, \mathcal{Z}, \rho\right)\right\} n=1, \cdots, N$, one at each energy. Thus, energetic photons interacting with the object (material) are attenuated according to each associated attenuation coefficient. An alternate representation incorporates the discretization of the object into voxels through $C T$-reconstruction followed by expressing transport as a set of coupled differential/difference equations [10]. State-space methods can be applied in either of these representations. Here we concentrate on the lumped model, since it is the simplest to comprehend.

2) X-ray Detector Measurements: Next let us consider an $\mathrm{X}$-ray beam or equivalently ray path consisting of polyenergetic photons irradiating the material and measured by a detector element, say the $\ell$-th. We choose to use a scintillation measurement system composed of a crystal converting the x-ray radiation to visible light photons coupled to a photo diode converting the photon energy to electrical charge/current. With this measurement system, the detector is related to the photon intensity through a set of energy dependent conversion constants, that is, for the $\ell$-th detector element and a set of $N$-energies, we have that

$$
d_{\ell}(\epsilon, x)=\underline{c}_{\ell}^{T}(\epsilon, \triangle \epsilon) \underline{\mathcal{I}}_{\ell}(\epsilon, x)=\sum_{n=1}^{N} c_{\ell}\left(\epsilon_{n}\right) \mathcal{I}_{\ell}\left(\epsilon_{n}, x\right)
$$

where $\underline{c}_{\ell}, \underline{\mathcal{I}}_{\ell} \in \mathcal{R}^{N \times 1}$ and $\underline{c}_{\ell}$ is an energy dependent vector of scintillation scale factors converting photon energy-to-charge (current).

For acquisition speed, an array of $L$-elements is normally applied, that is, expanding over $\ell$, we obtain the detector measurement model as

$$
\left[\begin{array}{c}
d_{1}(\epsilon, x) \\
--- \\
\vdots \\
--- \\
d_{L}(\epsilon, x)
\end{array}\right]=\left[\begin{array}{c}
\underline{c}_{1}^{T}(\epsilon, \Delta \epsilon) \\
--- \\
\vdots \\
--- \\
\underline{c}_{L}^{T}(\epsilon, \Delta \epsilon)
\end{array}\right] \underline{\mathcal{I}}(\epsilon, x)
$$

which can also be expressed simply as

$$
\underline{\mathbf{d}}(\epsilon, x)=\mathbf{C}(\epsilon, \triangle \epsilon) \underline{\mathcal{I}}(\epsilon, x)
$$

where we have the detector measurement vector, $\underline{\mathbf{d}} \in \mathcal{R}^{L \times 1}$, the measurement system matrix, $\mathbf{C} \in \mathcal{R}^{L \times N}$, and the corresponding state intensity vector $\underline{\mathcal{I}} \in \mathcal{R}^{N \times 1}$.

Thus, the overall physics-based state-space description (deterministic) of the $\mathrm{x}$-ray transmission and detector system is summarized by combining the relations of Eqs. 5 and 10 for each ray and detector element as

$$
\begin{aligned}
\frac{d}{d x} \underline{\mathcal{I}}(\epsilon, x) & =\mathbf{A}(\epsilon ; \theta) \underline{\mathcal{I}}(\epsilon, x) \\
\underline{\mathbf{d}}(\epsilon, x) & =\mathbf{C}(\epsilon, \triangle \epsilon) \underline{\mathcal{I}}(\epsilon, x)
\end{aligned}
$$

where the $N$-state intensity vector by $\underline{\mathcal{I}}$, the $N \times N$ system matrix is defined by $\mathbf{A}(\epsilon ; \theta):=\operatorname{diag}\left[-\mu\left(\epsilon_{1} ; \theta\right), \cdots,-\mu\left(\epsilon_{N} ; \theta\right)\right]$ and the material parameter set by $\theta:=\{\mathcal{Z}, \rho\}$.

We summarize the development of the state-space representations in Fig. 2a. Here we see the evolution of the photon intensity proportional to the detector output current. Initially, the polyenergetic source represented by the composition of a set of monoenergetic sources $(\underline{\mathcal{I}}(\epsilon, x))$ irradiating the object. The transmitted output $(\underline{\mathcal{I}}(\epsilon, x))$ is collected by the detector array, integrated, producing a set of output currents proportional to the transmitted photon energies $(\underline{\mathbf{d}}(\epsilon, x))$ used as input to image the object attenuation. The voxel $(C T)$ representation is also included in Fig. 2b for completeness.

This completes the overall state-space description of the $\mathrm{X}$-ray transmission physics and detector (array) measurement system. Note also that a stochastic description incorporates a variety of noise and parameter uncertainty processes that we shall discuss subsequently in later sections. 

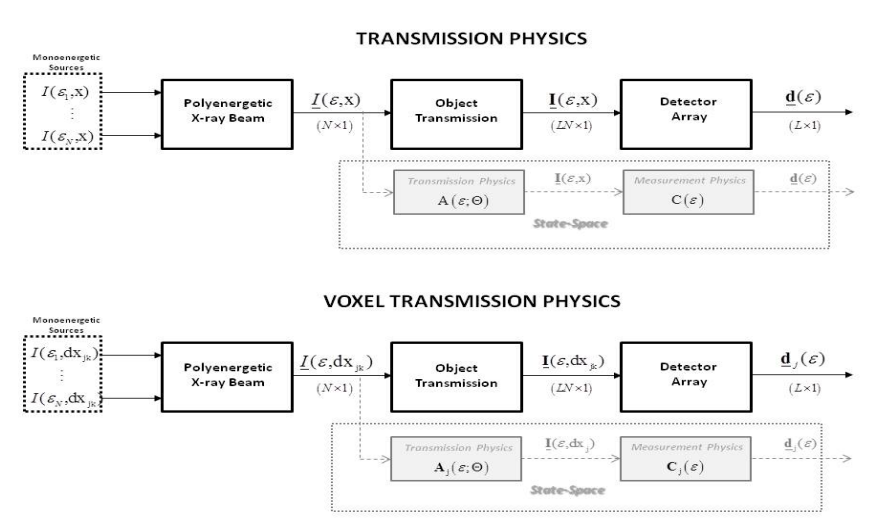

Fig. 2. X-ray transmission: (a) Transmission physics with "lumped" statespace model (dashed). (b) Voxel physics for $j$-th ray with state-space model (dashed).

\section{X-Ray State-Space Simulation}

In this section we briefly discuss the development of a statespace simulator capable of synthesizing X-ray detector outputs for generating noisy detector data. The approach involves discretizing the solution using the state transition matrix which is a matrix exponential [9] and generating data for an object.

The basic deterministic state-space model of Eq. 11 can easily be extended to a stochastic representation assuming additive, zero-mean, Gaussian noise resulting in the GaussMarkov state-space model either given by the differential equation of Eq. 11 or equivalently in terms of the state transition matrix of Eq. 7. The system of equations may be discretized to obtain a sampled data representation [9] resulting in the following discrete Gauss-Markov model given by

$$
\underline{\mathcal{I}}\left(\epsilon, x_{k}\right)=\mathcal{T}\left(\epsilon, \triangle x_{k}\right) \underline{\mathcal{I}}\left(\epsilon, x_{k-1}\right)+\triangle x_{k} \mathcal{T}\left(\epsilon, \triangle x_{k}\right) \underline{\mathbf{w}}\left(x_{k-1}\right)
$$

with corresponding measurement (detector array) model

$$
\underline{\mathbf{d}}\left(\epsilon, x_{k}\right)=\mathbf{C}(e, \triangle \epsilon) \underline{\mathcal{I}}\left(\epsilon, x_{k-1}\right)+\underline{v}\left(x_{k}\right)
$$

for $N_{\mathcal{I}}$-intensity (state) and white, Gaussian process uncertainty/noise vectors, $\underline{\mathcal{I}}, \underline{\mathbf{w}}$ with $w \sim \mathcal{N}\left(0, R_{w w}\right)$. Here $\underline{\mathbf{d}}$ the $N_{d}$-measurement (detector array) with corresponding white, Gaussian uncertainty/noise (electronic) vector, $\underline{v}$ for $v \sim \mathcal{N}\left(0, R_{v v}\right)$ along with the corresponding $N_{d} \times N_{\mathcal{I}}$ measurement matrix, $\mathbf{C}$. The incremental path length is specified by $\triangle x_{k}:=x_{k}-x_{k-1}$ with $\left(N_{\mathcal{I}} \times N_{\mathcal{I}}\right)$ state transition matrix, $\mathcal{T}$

$$
\mathcal{T}\left(\epsilon, \triangle x_{k}\right)=e^{\mathbf{A}(\epsilon ; \theta) \triangle x_{k}}
$$

for $\mathbf{A}(\epsilon ; \theta)=\operatorname{diag}\left[-\mu\left(\epsilon_{1} ; \theta\right), \cdots,-\mu\left(\epsilon_{N_{d}} ; \theta\right)\right]$ the material attenuation coefficients and set of intrinsic material properties, $\{\theta\}$. In addition to the intensity and measurement models, the Gauss-Markov representation also propagates the associated statistics (state/measurements) means and variances which are used to construct uncertainty bounds around the synthesized data, that is, the mean state intensity is given by the relation

$$
\underline{\mathcal{M}}_{\mathcal{I}}\left(\epsilon, x_{k}\right)=\mathcal{T}\left(\epsilon, \triangle x_{k}\right) \underline{\mathcal{M}}_{\mathcal{I}}\left(\epsilon, x_{k-1}\right)
$$

along with the mean detector measurement

$$
\underline{\mathcal{M}}_{\mathbf{d}}\left(\epsilon, x_{k}\right)=\mathbf{C}(\epsilon, \Delta \epsilon) \underline{\mathcal{M}}_{\mathcal{I}}\left(\epsilon, x_{k}\right)
$$

The corresponding state and measurement covariances are given by

$$
\mathcal{P}_{\mathcal{I}}\left(x_{k}\right)=\mathcal{T}\left(\epsilon, \triangle x_{k}\right) \mathcal{P}_{\mathcal{I}}\left(x_{k-1}\right) \mathcal{T}^{T}\left(\epsilon, \triangle x_{k}\right)+\mathbf{R}_{w w}\left(x_{k-1}\right)
$$

$$
\mathcal{R}_{\mathbf{d d}}\left(x_{k}\right)=\mathbf{C}(\epsilon, \triangle \epsilon) \mathcal{P}_{\mathcal{I}}\left(x_{k-1}\right) \mathbf{C}^{T}(\epsilon, \triangle \epsilon)+\mathbf{R}_{v v}\left(x_{k}\right)
$$

To summarize this "lumped" model representation, the discrete Gauss-Markov model characterizes the photon transport through a homogeneous object irradiated by a polyenergetic source with uncertainties approximated by additive zero-mean, white Gaussian sequences both in intensity (process) and detector intensity measurements (measurement). Next we develop the simulator and demonstrate some of its results.

In this problem we assume that an object or item has been isolated and extracted from a $C T$-image resulting in an attenuation coefficient sequence (ACS). From this sequence a set of average attenuation coefficients is obtained at each known source energy and represented by $\left\{\mu\left(\epsilon_{n}, \theta\right\} n=1, \cdots, N\right.$. We developed a simple transport simulator based on the lumped model and the state-space framework to synthesize a threat object composed of ammonium nitrate (NH4NO3) material characterized uniquely by its set of energy dependent attenuation coefficients obtained from the tables [1] based on a polyenergetic source and detector bands at $30-160 \mathrm{KeV}$ incremented every $10 \mathrm{KeV}$. The transport is calculated every $\triangle x=100 \mathrm{~mm}$. We assume that an x-ray beam (ray) at $0^{\circ}$ passes through the object (no clutter) and is measured by an 8-element scintillation detector array (see Fig. 3). We also assume that uncertainties propagate and are captured by the model discussed above with intensity (process) uncertainty of covariance $R_{w w}=25$ and detector measurement noise of covariance $R_{v v}=10^{7}$. The scanner volume can be considered a $1 \mathrm{~m}$ spherical volume. The problem parameters are summarized in Table I. We have $N_{\mathcal{I}}=14$ states (and average attenuation coefficients) with $N_{d}=8$ elements in the detector array. The simulation was performed for a single beam (ray) propagating through the object at a total path length of $100 \mathrm{~cm}$ (scan volume) with the object of cross-section $6 \mathrm{~cm}$ located $30 \mathrm{~cm}$ from the source. 


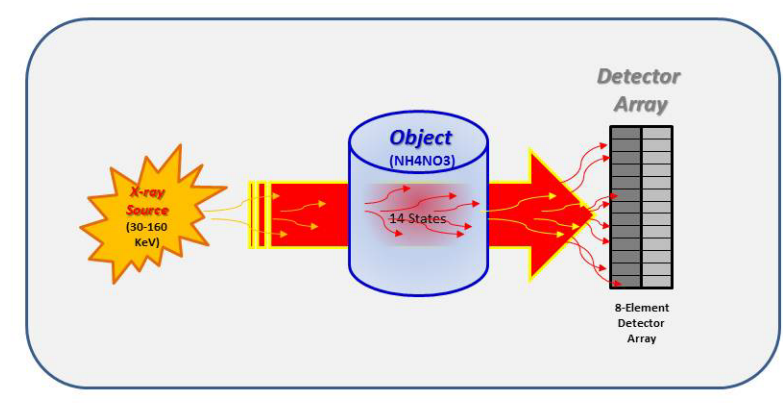

Fig. 3. X-ray transmission physics state-space lumped-model simulation of object irradiated by a polyenergetic source $(30-160 \mathrm{KeV})$ characterized by a set of attenuation coefficients of ammonium-nitrate material and 8-element scintillation detector array.

Table I. Transport Physics Simulation (Ammonium Nitrate).

\begin{tabular}{||c|c||}
\hline \hline Energy (KeV) & Atten. Coefficients \\
\hline 30 & 0.95388 \\
\hline 40 & 0.68456 \\
\hline 50 & 0.58017 \\
\hline 60 & 0.52641 \\
\hline 70 & 0.49307 \\
\hline 80 & 0.47019 \\
\hline 90 & 0.45164 \\
\hline 100 & 0.43706 \\
\hline 110 & 0.42389 \\
\hline 120 & 0.41288 \\
\hline 130 & 0.40292 \\
\hline 140 & 0.39344 \\
\hline 150 & 0.38511 \\
\hline 160 & 0.37761 \\
\hline
\end{tabular}

The results of the simulation are shown in Figs. 4 and 5. As expected, the photons are at the initial intensity $\mathcal{I}\left(\epsilon_{o}, x_{o}\right)$ and then drop off exponentially based on their respective attenuation coefficients and detector measurements as they interact with the material (ammonium-nitrate) with the path length incremented in $\triangle x=0.1 \mathrm{~cm}$ steps. Note that in reality the detector array provide only a single snapshot of integrated intensity at a given $x$-location providing a measured projection [2]. Here we applied additive Gaussian noise to approximate the uncertainty of the transported photons as well as measurement uncertainty (electronic noise) at the detector array. Later in Sec. 4, we will discuss the application of a physics-based processor capable of enhancing the intensity and extracting the states.

The resulting intensity (states) at a variety of array locations (along the x-axis) is shown in the surface plot of Fig. 4.

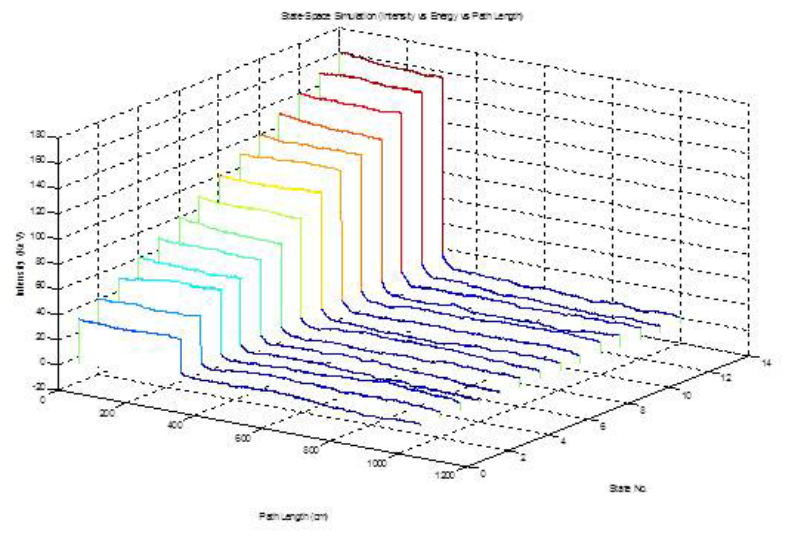

Fig. 4. X-ray transmission physics state-space simulation: Surface representation of state intensities with zero-mean, additive Gaussian noise (process).

We note the differing exponential decay rates along with the additive Gaussian intensity noise (process). The noisy detector measurement surface is shown in Fig. 5 with similar characteristics. This completes the lumped model simulations which will be used as input to the sequential detector algorithm.

\section{Sequential Bayesian Threat Detection}

Detection of threats residing in a variety of containers is essentially a binary detection problem. Here we investigate a solution to this decision problem to detect the presence of threat materials using $\mathrm{x}$-ray radiation as the primary modality (e.g. pot scanners) to irradiate each object and assess its status-threat or non-threat. Therefore, we define the problem in terms of a binary hypothesis test. From the scanner we use detector outputs directly available from each of the array elements, that is, the binary threat detection problem is based on testing the hypotheses

$$
\begin{aligned}
& \mathcal{H}_{0}: \underline{\mathbf{d}}\left(\epsilon, x_{k}\right)=\mathbf{C}(\epsilon, \Delta \epsilon) \underline{\mathcal{I}}\left(\epsilon, x_{k} ; \theta\right)+v\left(x_{k}\right) \quad \text { [NON-THREAT] } \\
& \mathcal{H}_{1}: \underline{\mathbf{d}}\left(\epsilon, x_{k}\right)=\mathbf{C}(\epsilon, \triangle \epsilon) \underline{\mathcal{I}}\left(\epsilon, x_{k} ; \theta_{t}\right)+v\left(x_{k}\right) \quad[\text { THREAT] (19) }
\end{aligned}
$$

where

$\underline{\mathbf{d}}$ is the $N_{d}$-element detector array

$\underline{\mathbf{C}}$ is the $\left(N_{d} \times N_{\mathcal{I}}\right)$ - energy measurement matrix

$\underline{\mathcal{I}}$ is the $N_{\mathcal{I}}$ - energy dependent intensities (states) 


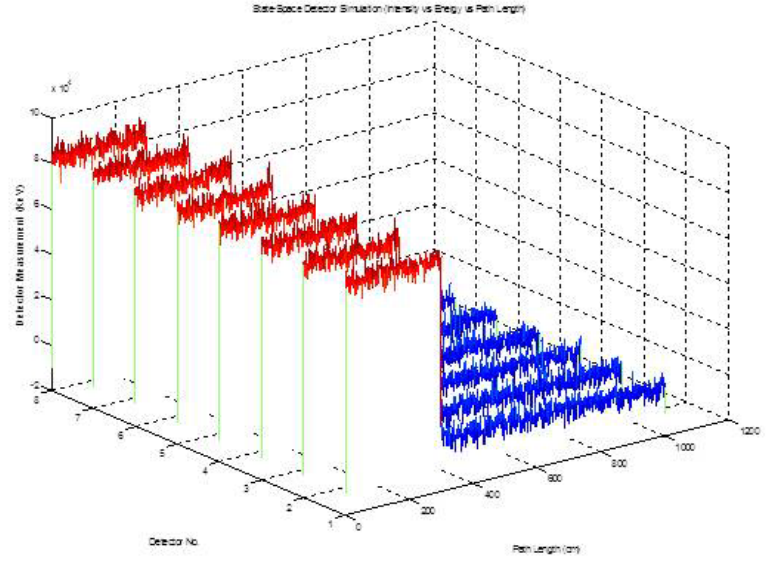

Fig. 5. X-ray transmission physics detector simulation: Surface representation of detector measurements with zero-mean, additive Gaussian noise (measurement).

$\underline{\mathbf{v}}$ is the $N_{d}$ - white Gaussian uncertainty/noise vector

$\underline{\theta}$ is the set of material properties (object and target)

$x_{k}$ is the length along the ray

The optimal solution to this decision problem evolves directly from the Neyman-Pearson theorem [18]-[19] maximizing the detection probability for a fixed false-alarm probability resulting in the likelihood-ratio decision function given by defined by

$$
\mathcal{L}\left[\mathcal{D}_{K}\right]:=\frac{\operatorname{Pr}\left[\mathcal{D}_{K} \mid \mathcal{H}_{1}\right]}{\operatorname{Pr}\left[\mathcal{D}_{K} \mid \mathcal{H}_{0}\right]}
$$

where $\mathcal{D}_{K}:=\left\{\underline{\mathbf{d}}\left(\epsilon, x_{1}\right), \cdots, \underline{\mathbf{d}}\left(\epsilon, x_{K}\right)\right\}$ with the hypothesis test defined by

$$
\begin{aligned}
& \mathcal{H}_{1} \\
\mathcal{L}\left[\mathcal{D}_{K}\right] & > \\
& <T \\
& \mathcal{H}_{0}
\end{aligned}
$$

for threshold $T$. This expression implies a "batch" decision, that is, we gather the $K$-detector array measurements, calculate the likelihood of Eq. 21 over the entire batch of data and compare it to the threshold to make the decision.
Expanding the likelihood ratio for each detector output, we obtain

$$
\mathcal{L}\left[\mathcal{D}_{K}\right]=\frac{\operatorname{Pr}\left[\underline{\mathbf{d}}\left(\epsilon, x_{1}\right), \cdots, \underline{\mathbf{d}}\left(\epsilon, x_{K}\right) \mid \mathcal{H}_{1}\right]}{\operatorname{Pr}\left[\underline{\mathbf{d}}\left(\epsilon, x_{1}\right), \cdots, \underline{\mathbf{d}}\left(\epsilon, x_{K}\right) \mid \mathcal{H}_{0}\right]}
$$

From the Bayes' rule [16], we have that

$$
\begin{aligned}
\operatorname{Pr}\left[\mathcal{D}_{K} \mid \mathcal{H}_{\ell}\right] & =\operatorname{Pr}\left[\underline{\mathbf{d}}\left(\epsilon, x_{1}\right), \cdots, \underline{\mathbf{d}}\left(\epsilon, x_{K}\right) \mid \mathcal{H}_{\ell}\right] \\
& =\operatorname{Pr}\left[\mathbf{d}\left(\epsilon, x_{K}\right), \mathcal{D}_{K-1} \mid \mathcal{H}_{\ell}\right]
\end{aligned}
$$

and

$$
\begin{aligned}
& \operatorname{Pr}\left[\underline{\mathbf{d}}\left(\epsilon, x_{K}\right), \mathcal{D}_{K-1} \mid \mathcal{H}_{\ell}\right] \\
= & \operatorname{Pr}\left[\underline{\mathbf{d}}\left(\epsilon, x_{k}\right) \mid \mathcal{D}_{K-1}, \mathcal{H}_{\ell}\right] \times \operatorname{Pr}\left[\mathcal{D}_{K-1} \mid \mathcal{H}_{\ell}\right] ; \ell=0,1
\end{aligned}
$$

Substituting these expressions into the likelihood ratio above, replacing $K \rightarrow k$ and grouping, we obtain

$$
\mathcal{L}\left[\mathcal{D}_{k}\right]=\left[\frac{\operatorname{Pr}\left[\mathcal{D}_{k-1} \mid \mathcal{H}_{1}\right]}{\operatorname{Pr}\left[\mathcal{D}_{k-1} \mid \mathcal{H}_{0}\right]}\right] \times \frac{\operatorname{Pr}\left[\underline{\mathbf{d}}\left(\epsilon, x_{k}\right) \mid \mathcal{D}_{k-1}, \mathcal{H}_{1}\right]}{\operatorname{Pr}\left[\underline{\mathbf{d}}\left(\epsilon, x_{k}\right) \mid \mathcal{D}_{k-1}, \mathcal{H}_{0}\right]}
$$

and the recursion or equivalently sequential likelihood ratio for the $k$-th detector output follows as [19], [20]

$\mathcal{L}\left[\mathcal{D}_{k}\right]=\mathcal{L}\left[\mathcal{D}_{k-1}\right] \times \frac{\operatorname{Pr}\left[\underline{\mathbf{d}}\left(\epsilon, x_{k}\right) \mid \mathcal{D}_{k-1}, \mathcal{H}_{1}\right]}{\operatorname{Pr}\left[\underline{\mathbf{d}}\left(\epsilon, x_{k}\right) \mid \mathcal{D}_{k-1}, \mathcal{H}_{0}\right]} ; \quad k=0,1, \cdots, K$

with $\operatorname{Pr}\left[\underline{\mathbf{d}}\left(\epsilon, x_{0}\right) \mid \mathcal{D}_{-1}, \mathcal{H}_{\ell}\right]=\operatorname{Pr}\left[\underline{\mathbf{d}}\left(\epsilon, x_{1}\right) \mid \mathcal{H}_{\ell}\right]$, the prior under each hypothesis.

Anticipating the exponential family of distributions [13], we take logarithms to obtain the sequential log-likelihood ratio (decision function) $\Lambda\left[\mathcal{D}_{k}\right]$, that is,

$$
\begin{aligned}
\Lambda\left[\mathcal{D}_{k}\right] & =\Lambda\left[\mathcal{D}_{k-1}\right]+\ln \operatorname{Pr}\left[\underline{\mathbf{d}}\left(\epsilon, x_{k}\right) \mid \mathcal{D}_{k-1}, H_{1}\right] \\
& -\ln \operatorname{Pr}\left[\underline{\mathbf{d}}\left(\epsilon, x_{k}\right) \mid \mathcal{D}_{k-1}, H_{0}\right]
\end{aligned}
$$

Therefore, the Wald sequential probability-ratio test is [19], [20]

$$
\begin{array}{lll}
\Lambda\left[\mathcal{D}_{k}\right] & >\ln T_{1}(k) & \text { Accept } H_{1} \\
\ln T_{0}(k) \leq \Lambda\left[\mathcal{D}_{k}\right] \leq \ln T_{1}(k) & \text { Continue } \\
\Lambda\left[\mathcal{D}_{k}\right] & <\ln T_{0}(k) & \text { Accept } H_{0}
\end{array}
$$

where the thresholds are specified in terms of the false alarm $\left(\mathrm{P}_{F A}\right)$ and miss $\left(\mathrm{P}_{M}\right)$ probabilities (from ROC curve) as

$$
T_{0}(k)=\frac{\mathrm{P}_{M}(k)}{\mathrm{P}_{F A}(k)} ; \quad T_{1}(k)=\frac{1-\mathrm{P}_{M}(k)}{\mathrm{P}_{F A}(k)}
$$

The sequential Bayesian detector operation is illustrated in Fig. 6 where we observe the updated decision function at each step along with the upper and lower decision thresholds based on the desired operating point (from a ROC curve). 


$$
\mathcal{T}\left(\epsilon, \triangle x_{k}\right)=e^{\mathbf{A}(\epsilon ; \theta) \triangle x_{k}}
$$

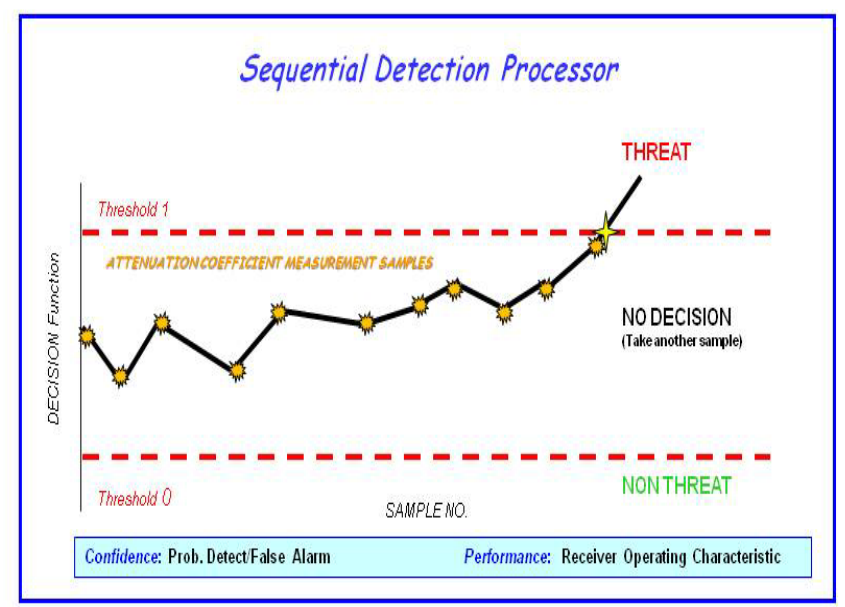

Fig. 6. Sequential Bayesian processor operation. At each measurement the attenuation coefficients are extracted and a threat or non-threat or take-anothersample is declared.

\section{A. Gaussian Case}

In this section, we apply the Gaussian assumption to the unknown distributions and develop the sequential Bayesian processor. Thus, for our $\mathrm{x}$-ray material detection problem, we must specify the required probabilities for each hypothesis which implies that we must incorporate the underlying transport physics (Beer's law) developed previously. Incorporating uncertainty and noise into the representation, the following Gauss-Markov model evolves directly from the state-space models as

$$
\underline{\mathcal{I}}\left(\epsilon, x_{k}\right)=\mathcal{T}\left(\epsilon, \triangle x_{k}\right) \underline{\mathcal{I}}\left(\epsilon, x_{k-1}\right)+\triangle x_{k} \mathcal{T}\left(\epsilon, \triangle x_{k}\right) \underline{\mathbf{w}}\left(x_{k-1}\right)
$$

with corresponding measurement (detector array) model

$$
\underline{\mathbf{d}}\left(\epsilon, x_{k}\right)=\mathbf{C}(e, \triangle \epsilon) \underline{\mathcal{I}}\left(\epsilon, x_{k-1}\right)+\underline{v}\left(x_{k}\right)
$$

with $N_{\mathcal{I}}$-intensity (state) and white, Gaussian process uncertainty/noise vectors, $\underline{\mathcal{I}}, \underline{\mathbf{w}}$ with $w \sim \mathcal{N}\left(0, R_{w w}\right)$. Here $\underline{\mathbf{d}}$ the $N_{d}$-measurement (detector array) with corresponding white, Gaussian uncertainty/noise (electronic) vector, $\underline{v}$ for $v \sim \mathcal{N}\left(0, R_{v v}\right)$ along with the corresponding $N_{d} \times N_{\mathcal{I}}$ measurement matrix, C. The path length is specified by $\triangle x_{k}:=x_{k}-x_{k-1}$ with $\left(N_{\mathcal{I}} \times N_{\mathcal{I}}\right)$ state transition matrix, $\mathcal{T}$ for $\mathbf{A}(\epsilon ; \theta)=\operatorname{diag}\left[-\mu\left(\epsilon_{1} ; \theta\right), \cdots,-\mu\left(\epsilon_{N} ; \theta\right)\right]$ the material attenuation coefficients and set of intrinsic material properties, $\{\theta\}$.

With this representation of $\mathrm{x}$-ray transport, it is possible to specify the binary decision problem

$$
\begin{aligned}
& \mathcal{H}_{0}: \underline{\mathbf{d}}\left(\epsilon, x_{k}\right)=\mathbf{C}(\epsilon, \Delta \epsilon) \underline{\hat{\mathcal{I}}}\left(\epsilon, x_{k} ; \theta\right)+v\left(x_{k}\right) \text { [NON-THREAT] } \\
& \mathcal{H}_{1}: \underline{\mathbf{d}}\left(\epsilon, x_{k}\right)=\mathbf{C}(\epsilon, \triangle \epsilon) \underline{\mathcal{I}}\left(\epsilon, x_{k} ; \theta_{t}\right)+v\left(x_{k}\right)[\text { THREAT] (34) }
\end{aligned}
$$

by the Gaussian distributions

$$
\begin{aligned}
& \operatorname{Pr}\left[\underline{\mathbf{d}}\left(\epsilon, x_{k}\right) \mid \mathcal{D}_{k-1} ; H_{0}\right]=\mathcal{N}\left(\underline{\hat{\mathbf{d}}}\left(x_{k} \mid x_{k-1}\right), \mathbf{R}_{e e}\right) \\
& \operatorname{Pr}\left[\underline{\mathbf{d}}\left(\epsilon, x_{k}\right) \mid \mathcal{D}_{k-1} ; H_{1}\right]=\mathcal{N}\left(\underline{\mathbf{d}}\left(\epsilon, x_{k} ; \theta_{t}\right), \mathbf{R}_{d d}\right)
\end{aligned}
$$

or

$$
\begin{aligned}
& \operatorname{Pr}\left[\underline{\mathbf{d}}\left(\epsilon, x_{k}\right) \mid \mathcal{D}_{k-1} ; H_{0}\right]=(2 \pi)^{-N_{d} / 2}\left|\mathbf{R}_{e e}\right|^{-1 / 2} \times \\
& e^{-\frac{1}{2}\left(\underline{\mathbf{d}}\left(\epsilon, x_{k}\right)-\underline{\mathbf{d}}\left(x_{k} \mid x_{k-1}\right)\right)^{T} \mathbf{R}_{e e}^{-1}\left(\underline{\mathbf{d}}\left(\epsilon, x_{k}\right)-\underline{\mathbf{d}}\left(x_{k} \mid x_{k-1}\right)\right)} \\
& \operatorname{Pr}\left[\underline{\mathbf{d}}\left(\epsilon, x_{k}\right) \mid \mathcal{D}_{k-1} ; H_{1}\right]=(2 \pi)^{-N_{d} / 2}\left|\mathbf{R}_{d d}\right|^{-1 / 2} \times \\
& e^{-\frac{1}{2}\left(\underline{\mathbf{d}}\left(\epsilon, x_{k}\right)-\underline{\mathbf{d}}\left(\epsilon, x_{k} ; \theta_{t}\right)\right)^{T} \mathbf{R}_{d d}^{-1}\left(\underline{\mathbf{d}}\left(\epsilon, x_{k}\right)-\underline{\mathbf{d}}\left(\epsilon, x_{k} ; \theta_{t}\right)\right)}
\end{aligned}
$$

Taking logarithms of these distributions and inserting them into the log-likelihood expression of Eq. 28, we obtain

$$
\begin{aligned}
& \Lambda\left[\mathcal{D}_{k}\right]=\Lambda\left[\mathcal{D}_{k-1}\right]-\ln \left((2 \pi)^{-N_{d} / 2}\left|\mathbf{R}_{d d}\right|^{-1 / 2}\right) \\
& -\frac{1}{2}\left(\underline{\mathbf{d}}\left(\epsilon, x_{k}\right)-\underline{\mathbf{d}}\left(\epsilon, x_{k} ; \theta_{t}\right)\right)^{T} \mathbf{R}_{d d}^{-1}\left(\underline{\mathbf{d}}\left(\epsilon, x_{k}\right)-\underline{\mathbf{d}}\left(\epsilon, x_{k} ; \theta_{t}\right)\right) \\
& +\ln \left((2 \pi)^{-N_{d} / 2}\left|\mathbf{R}_{e e}\right|^{-1 / 2}\right) \\
& +\frac{1}{2}\left(\underline{\mathbf{d}}\left(\epsilon, x_{k}\right)-\underline{\hat{\mathbf{d}}}\left(x_{k} \mid x_{k-1}\right)\right)^{T} \mathbf{R}_{e e}^{-1}\left(\underline{\mathbf{d}}\left(\epsilon, x_{k}\right)-\underline{\hat{\mathbf{d}}}\left(x_{k} \mid x_{k-1}\right)\right)
\end{aligned}
$$

Since the underlying physics-based models are assumed Gauss-Markov, then we know that the optimal solution to the linear estimation of the detector array measurement is the conditional mean with sequential solution provided by the well-known Kalman filter [9]. Therefore, we have that $\underline{\hat{\mathbf{d}}}\left(x_{k} \mid x_{k-1}\right)=E\left\{\underline{\mathbf{d}}\left(\epsilon, x_{k}\right) \mid \mathcal{D}_{k-1}\right\}$ the conditional mean at path length $x_{k}$ based on past path lengths up to $x_{k-1}$ along with conditional covariance, $R_{e e}\left(x_{k}\right)=\operatorname{cov}\left(\underline{\mathbf{e}}\left(x_{k}\right)\right)$-the innovations covariance provided by the sequential algorithm, that is, 


$$
\begin{aligned}
\hat{\underline{\mathcal{I}}}_{\epsilon}\left(x_{k} \mid x_{k-1}\right) & =\mathcal{T}\left(\epsilon, \triangle x_{k} ; \theta\right) \hat{\mathcal{I}}_{\epsilon}\left(x_{k-1} \mid x_{k-1}\right) \text { [Pred. State] } \\
\underline{\hat{\mathbf{d}}}\left(x_{k} \mid x_{k-1}\right) & =\mathbf{C}(\epsilon, \triangle \epsilon) \hat{\mathcal{I}}_{\epsilon}\left(x_{k} \mid x_{k-1}\right)[\text { Pred. Measurement] } \\
\tilde{\mathbf{P}}_{\mathcal{I}}\left(x_{k} \mid x_{k-1}\right) & =\mathcal{T}\left(\epsilon, \triangle x_{k} ; \theta\right) \tilde{\mathbf{P}}_{\mathcal{I}}\left(x_{k-1} \mid x_{k-1}\right) \mathcal{T}\left(\epsilon, \triangle x_{k} ; \theta\right)^{T} \\
& +\mathbf{R}_{w w}\left(x_{k-1}\right)[\text { Pred. Cov.] } \\
\underline{\mathbf{e}}\left(x_{k}\right) & \left.=\underline{\mathbf{d}}\left(\epsilon, x_{k}\right)-\hat{\underline{\mathbf{d}}}\left(x_{k} \mid x_{k-1}\right)\right)[\text { Innovations] } \\
\mathbf{R}_{e e}\left(x_{k}\right) & =\mathbf{C}(\epsilon, \triangle \epsilon) \tilde{\mathbf{P}}_{\mathcal{I}}\left(x_{k} \mid x_{k-1}\right) \mathbf{C}^{T}(\epsilon, \triangle \epsilon) \\
& +\mathbf{R}_{v v}\left(x_{k}\right)[\text { Innovations Cov.] }
\end{aligned}
$$

Here we ignore the update terms in the algorithm (for details see [9]).

An expression for the "target" or "true" distribution can be specified using the true (target) parameters from the available tables [1] for

$$
\underline{\mathcal{M}}_{\mathcal{I}}\left(\epsilon, x_{k}\right)=\mathcal{T}\left(\epsilon, \triangle x_{k}\right) \underline{\mathcal{M}}_{\mathcal{I}}\left(\epsilon, x_{k}\right)
$$

along with the mean detector measurement

$$
\underline{\mathcal{M}}_{\mathbf{d}}\left(\epsilon, x_{k}\right)=\mathbf{C}(\epsilon, \triangle \epsilon) \underline{\mathcal{M}}_{\mathcal{I}}\left(\epsilon, x_{k-1}\right)
$$

The corresponding state and measurement mean and covariances are given by Eqs. 15-18, that is,

$$
\begin{aligned}
\underline{\mathbf{d}}\left(\epsilon, x_{k} ; \theta_{t}\right) & \leftrightarrow \underline{\mathcal{M}}_{\mathbf{d}}\left(\epsilon, x_{k}\right) \\
\mathbf{R}_{d d}\left(x_{k}\right) & \leftrightarrow \mathbf{C}(\epsilon, \Delta \epsilon) \mathcal{P}_{\mathcal{I}}\left(x_{k-1}\right) \mathbf{C}^{T}(\epsilon, \Delta \epsilon)+\mathbf{R}_{v v}\left(x_{k}\right)
\end{aligned}
$$

Substituting these expressions into the log-likelihood decision function, we obtain

$$
\begin{aligned}
& \Lambda\left[\mathcal{D}_{k}\right]=\Lambda\left[\mathcal{D}_{k-1}\right]+\kappa-\frac{1}{2}\left(\underline{\mathbf{d}}\left(\epsilon, x_{k}\right)-\underline{\mathcal{M}}_{\mathbf{d}}\left(\epsilon, x_{k}\right)\right)^{T} \mathbf{R}_{d d}^{-1} \\
& \left(x_{k}\right)\left(\underline{\mathbf{d}}\left(\epsilon, x_{k}\right)-\underline{\mathcal{M}}_{\mathbf{d}}\left(\epsilon, x_{k}\right)\right)+\frac{1}{2}\left(\underline{\mathbf{e}}^{T}\left(x_{k}\right) \mathbf{R}_{e e}^{-1}\left(x_{k}\right) \underline{\mathbf{e}}\left(x_{k}\right)\right)
\end{aligned}
$$

where $\kappa:=\frac{1}{2}\left(\ln \left|\mathbf{R}_{e e}\left(x_{k}\right)\right|-\ln \left|\mathbf{R}_{d d}\left(x_{k}\right)\right|\right)$.

Following the simulation of Sec. 3, we developed the physics-based processor to enhance the intensity states and detector measurements. The results are shown in Figs. 7 and 8. In Fig. 7 we observe the intensity (state) estimates clearly demonstrating the capability of the processor along with the enhanced measurements shown in Fig. 8. Clearly the noisy detector array measurements have been enhanced significantly compared to Fig. 5.

Finally to demonstrate the sequential Bayesian detection algorithm using the enhanced data provided by the Kalman filter (above), we applied it to the synthesized data with a lower signal-to-noise ratio $(S N R)$ with the resulting output shown in Fig. 9. We note the log-likelihood decision function and the associated bounds assuming a detection probability of

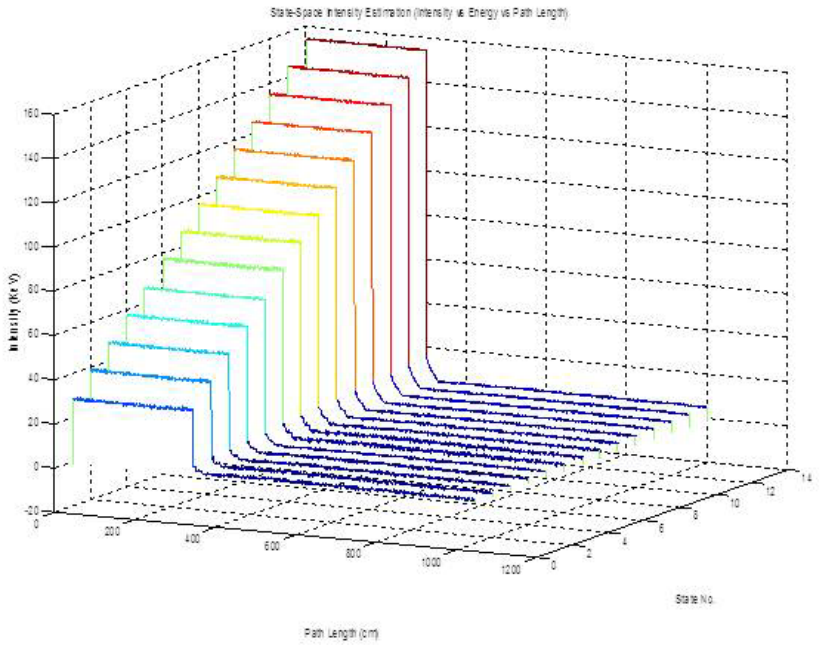

Fig. 7. Physics-based processor results: Intensity (state conditional mean) estimation.

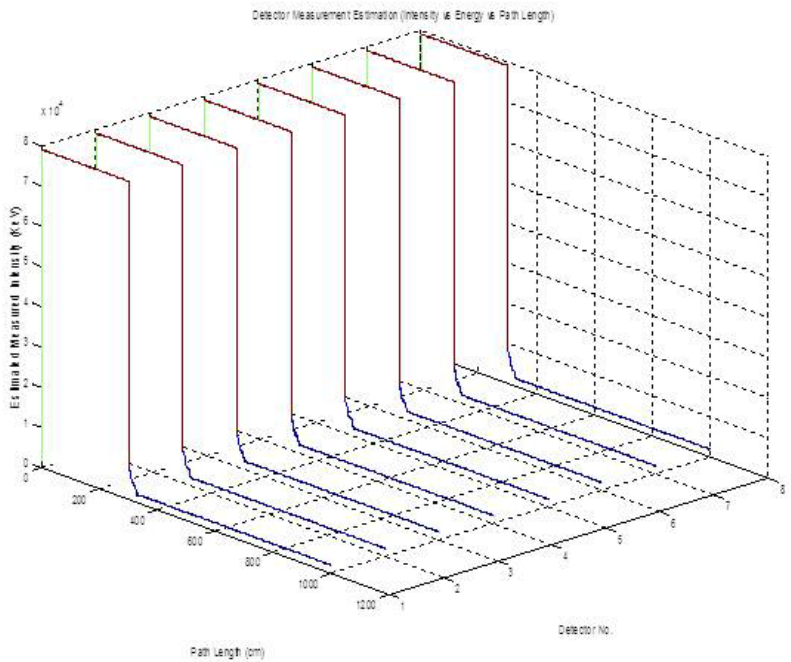

Fig. 8. Physics-based processor results: Detector measurement estimation. 


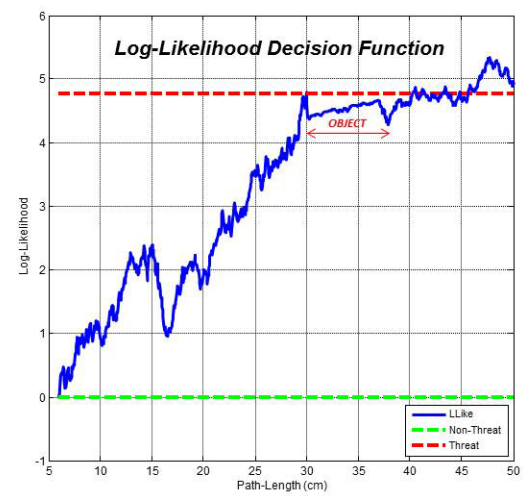

Fig. 9. Physics-based processor threat detection for Gaussian case: Loglikelihood ratio decision function (green) and thresholds for an assumed detection and false alarm probabilities of $95 \%$ and $5 \%$, respectively.

$95 \%$ and a false alarm probability of $5 \%$ which are used to calculate the upper and lower thresholds. The results for this case are expected with the log-likelihood function increasing until the detection threshold is crossed. Next we summarize our results and discuss future efforts.

\section{SUMMARY}

We have demonstrated that a physics-based sequential Bayesian processor [13] can be developed to provide a feasible solution to the $\mathrm{x}$-ray threat contraband detection problem by defining a target $\mathrm{x}$-ray item(s). Sequential techniques based on the state-space formulation [9] were developed and discussed demonstrating their ability to capture the $\mathrm{x}$-ray transport physics for a lumped transport model representation. The lumped approach was further investigated and an x-ray transport synthesizer was developed including uncertainties resulting in a Gauss-Markov state-space formulation. Synthesized data for an ammonium-nitrate explosive threat was then employed to investigate the performance of a sequential Bayesian detector incorporating a physics-based processor (Kalman filter [9]) for intensity measurement enhancement. The resulting log-likelihood detection technique was capable of performing a detection under the assumed detection and false alarm probabilities. Thus, the Bayesian approach enables the development of a physics-based detection algorithm capable of detected threat under restricted assumptions (Gaussian, etc.).

Future work involves the investigation of: (1) the lumped model approach to incorporate multiple beams at each angle; (2) the development and application of the sequential Bayesian detection algorithms directly for intensities (states); (3) the development of a voxel-based, state-space simulator, estimator and detector; (4) the application of the various processors to sophisticated simulation data; (5) the application to controlled experimental data (x-ray scanner); and (6) the application of the processor to cargo data.

\section{ACKNOWLEDGMENTS}

We would like to acknowledge the contributions of our nuclear experts, Drs. H. Martz, Jr. for his encouragement, guidance and tremendous insight in helping to direct our efforts, T. White, J. Smith, M. Aufderheide for their help on the transmission physics and measurement models and Drs. S. Azevedo and J. Kallman for their inputs on CT algorithms. This work performed under the auspices of the U.S. Department of Energy by Lawrence Livermore National Laboratory under Contract DE-AC52-07NA27344.

\section{REFERENCES}

[1] N. J. Carron, An Introduction to the Passage of Energetic Particles through Matter, New York, New York: Taylor and Francis Group, 2007.

[2] A. C. Kak and M. Slaney, Principles of Computerized Tomographic Imaging, New York, New York: IEEE Press, 1988.

[3] R. O. Duda, P.E. Hart and D. G. Stork Pattern Classification, $2^{\text {nd }}$ Ed., Hoboken, New Jersey: John Wiley \& Sons, 2001.

[4] K. Fukunaga, Statistical Pattern Recognition, $2^{\text {nd }}$ Ed., New York, New York: Academic Press, 1990.

[5] S. Theodoridis and K. Koutroumbas, Pattern Recognition, New York, New York: Academic Press, 1998.

[6] I. T. Nabney, NETLAB Algorithms for Pattern Recognition, New York, New York: Springer, 2003.

[7] C. M. Bishop, Pattern Recognition and Machine Learning, New York, New York: Springer, 2006.

[8] F. van der Heijden, R. P. Duin, D. de Ridder, and D. M. Tax, Classification, Parameter Estimation and State Estimation, Hoboken, New Jersey: John Wiley \& Sons, 2004.

[9] J. V. Candy, Model-Based Signal Processing, Hoboken, New Jersey: John Wiley/IEEE Press, 2006.

[10] J. V. Candy, Physics-Based X-ray Processing: A Feasibility Study, LLNL Report, LLNL-TR-605992, 2012

[11] B. D. Anderson and J. B. Moore, Optimal Filtering. Englewood Cliffs, New Jersey: Prentice-Hall, 1979.

[12] S. Haykin, Kalman Filtering and Neural Networks. (New York, John Wiley, 2001).

[13] J. V. Candy, Bayesian Signal Processing, Classical, Modern and Particle Filtering, Hoboken New Jersey: John Wiley/IEEE Press, 2009.

[14] S. Gulam Razul, W. J. Fitzgerald and C. Andrieu, Bayesian model selection and parameter estimation of nuclear emission spectra using RJMCMC, Nucl. Instrum. And Methods in Physics Res. A, Vol. 497, pp. 492-510, 2003.

[15] E. Barat and J. Heikkinen, An algorithm for nonparametric Bayesian estimation of Poisson intensity, Comput. Statist., Vol. 12, no. 3, 1997.

[16] A. Papoulis and S. Pillai, Probability, Random Variables and Stochastic Processes, 4th ed., New York, New York: McGraw-Hill, 2002.

[17] J. Liu, Monte Carlo Strategies in Scientific Computing, New York, New York: Springer-Verlag, 2001.

[18] D. Middleton, An Introduction to Statistical Communication Theory, New York, N.Y.: McGraw-Hill, 1960.

[19] A. Wald, "Sequential tests of statistical hypothesis," Ann. Math. Stat., 16, pp. 117-186, 1945.

[20] A. Wald, Sequential Analysis, New York, N.Y.: John Wiley, 1947 (Reprint Dover Publications, 1973). 